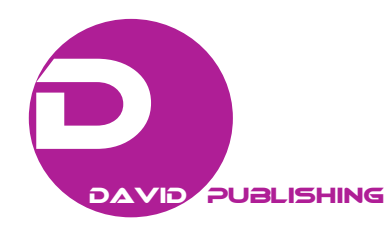

\title{
Application of MRWA RAP and ANRAM for Assessing Upgrades on the Great Eastern Highway
}

\author{
Jan Karpinski \\ Main Roads Western Australia
}

\begin{abstract}
A methodology has been previously developed to provide the required data for the iRAP Star Rating 7 model using data sourced from MRWA database information (corporate inventory) and road 8 condition information supplemented by other data sources. This is referred to as MRWA RAP to 9 distinguish from data collected in accordance with iRAP protocols which provides data for AusRAP. 10 The iRAP data is used by the Australian National Risk Assessment Model (ANRAM). This paper 11 describes the work done on updating MRWA RAP as well as the application of the ANRAM model 12 to assess proposed treatments on the Great Eastern Highway to improve road safety.
\end{abstract}

Key words: Road safety, highway, risk assessment.

\section{Background}

As documented in Karpinski [1], a methodology had been developed to assemble the required data for the iRAP Star Rating model using data sourced from MRWA database information (corporate inventory) and road condition information supplemented by other data sources. This data is referred to as MRWA RAP to avoid confusion between it and AusRAP. The focus of MRWA RAP to date has been to produce star ratings for vehicle occupants and to provide the necessary input data for the Australian National Risk Assessment Model (ANRAM). The iRAP dataset consists of 78 attributes which are required for every $100 \mathrm{~m}$ of the road being rated and are described in detail in iRAP $[2,3]$.

As the data used by MRWA RAP and AusRAP is different, the results of the star rating using both data sets will be different. Comparison of the differences and of both the inputs into the star rating model as well as the outputs can be found in Karpinski [1].

An assessment of proposed interim upgrades to the Great Eastern Highway from the intersection of the

Corresponding author: Jan Karpinski, consulting engineer; research fields: road, drainage, and flood protection.
Great Southern Highway to Mitchell Avenue intersection at Northam, a distance of approximately 40 $\mathrm{km}$, was made using ANRAM (V1.04 M) [4]. This paper describes the methodology used and the outcomes of the assessment.

\section{Method}

The original MRWA RAP data set was created in 2014 and had to be updated so that the ANRAM modelling reflected the current configuration and condition of the Great Eastern Highway to assist in the selection of appropriate treatments. Comparisons were made between the current inventory and condition versus the original data sets used. To simplify the process, iRAP codes (refer to iRAP [1] for details) were assigned to the data.

For some iRAP attributes there is a range of values, e.g. for paved shoulder width, a rating of 3 corresponds to narrow applies to a shoulder width $>=0 \mathrm{~m}$ to $<1.0 \mathrm{~m}$. Rather than trying to compare different shoulder widths, e.g. the previous sealed shoulder could have been $0.5 \mathrm{~m}$ and a revised seal width could be $0.8 \mathrm{~m}$, the iRAP coding was compared (in this case both have the same iRAP coding of 3 ). If, over the years, the paved shoulder had been increased in width but was still 
within the range, then the data was still current. Comparisons were done either using Excel macros or by using Beyond Compare.

As part of developing a concept design for the treatments, additional information such as intersection volumes and locations of driveways were obtained and the existing intersection layouts and horizontal geometry confirmed. These were checked against previous proxy methods and different geometry data sets were compared. A macro was written in Excel (Excel, like other Microsoft Office programs has VBA (Visual Basic for Applications) programing language) to identify changes between the original data set developed and the revised data set.

It was found that there were significant differences in the data sets. To assess the significance of these differences comparison of the iRAP star rating scores (SRS) which are used to assign star rating, was made using the Star Rating Demonstrator tool available through ViDA. ANRAM SRSs are based on iRAP SRS components but ANRAM assembles these SRS components in a different way which is used in the Crash Prediction Module. The basis of the SRS was a cross section that best represented the current state of Great Eastern Highway for a speed limit of $110 \mathrm{~km} / \mathrm{hr}$. The star rating score (SRS) of the base case was 12.75. The base case was a road with trees with 5 to $10 \mathrm{~m}$ from the edge line, narrow shoulders with audible edge lines, wide lanes, road condition, skid resistance and delineation in good condition and no intersections. The differences have been quoted as a $\%$ of the star rating score for the base case. Where there is a range of difference ratings obtained, the minimum and maximum changes were tested, and based on this analysis, a statement about the significance of the change attribute made.

A summary of all of the changes between the two data sets where there were more than 5 changes is shown in Tables 1 and 2. The tables show the difference in the iRAP codes (with the latest code shown first and the original code shown separated by a
"_). The number of times the differences occurred is shown in the column listed Count. Traffic volumes were also updated for the data set for all sections. A textual description in the change of the attribute coding is also shown.

A brief summary of the differences is given below.

Accesses: covers the addition of accesses not previously identified through corporate data, or identified as commercial rather than residential, or the removal of accesses.

Intersection Volumes: this covers where actual intersection traffic counts were found to be different from those that were assigned based on corporate data or on assumptions. The largest difference found in the rating was a change of 6 (corresponding to a volume range of 1-100) to 4 (volume range of 1,000-5,000) with most differences being a change of rating of 5 $(100$ to 1,000$)$ to $4(1,000-5,000)$. It should be noted that unless traffic volumes for the intersections are available, the iRAP coding manual recommends basing the traffic volume on the intersection layout in the absence of actual count data. Using the intersection layouts, it was found that the iRAP method understates the volumes for 9 of the intersections which had changed traffic volumes (those changed from 4 5).

Road condition and skid resistance: these had previously been set based on some alignment of the visual ratings versus road condition information (combination of rutting and roughness data) and skid resistance. This alignment is described in Karpinski [1] and the rating was updated using the latest corporate condition inventory.

Paved shoulder widths: there were a large number of differences in the paved shoulder widths with many being changed from narrow (sealed shoulder 0 to $<1 \mathrm{~m}$ ) to medium (sealed shoulder $>=1 \mathrm{~m}$ and $<2.4 \mathrm{~m}$ ).

Lighting: MRWA has no asset data for lighting in rural areas and the original data for lighting was extracted taken from crash history as part of the crash records indicate that there was lighting at the site. The extent of lighting was modified based on additional 
Table 1 Differences in the ANRAM Data Sets Part 1.

\begin{tabular}{|c|c|c|c|c|c|c|c|c|c|}
\hline Number of lanes & Count & Paved shoulder drivers side & Count & Paved shoulder passenger side & Count & Curvature & Count & Road condition & Count \\
\hline $1 \_5$ & 14 & $2 \_3$ & 63 & $2 \_1$ & 5 & $2 \_3$ & 6 & $1 \_2$ & 9 \\
\hline 5_1 & 11 & & & $2 \_3$ & 52 & & & & \\
\hline \multirow[t]{2}{*}{ Totals } & 25 & & 63 & & 57 & & 6 & & 9 \\
\hline & SRS & & SRS & & & & & & SRS \\
\hline From $1 / 2+1$ & 13.49 & From narrow / medium & 11.86 & \multirow{2}{*}{\multicolumn{2}{|c|}{$\begin{array}{l}\text { Both shoulder widths } \\
\text { changing }\end{array}$}} & From sharp / moderate & \multirow{2}{*}{22.32} & From good / medium & \multirow{2}{*}{15.14} \\
\hline & & From wide to medium & 11.41 & & & & & & \\
\hline Base SRS & 12.75 & \multicolumn{2}{|l|}{12.75} & & & \multicolumn{2}{|l|}{42.65} & \multicolumn{2}{|l|}{12.75} \\
\hline $\begin{array}{l}\text { Difference } \\
\text { SRS \% }\end{array}$ & $\mathrm{N} / \mathrm{A}$ & \multicolumn{2}{|l|}{-10.5} & & & \multicolumn{2}{|l|}{ N/A } & \multicolumn{2}{|l|}{$\mathrm{N} / \mathrm{A}$} \\
\hline Assessment & Significant & \multicolumn{2}{|l|}{ Significant } & & & \multicolumn{2}{|l|}{ Significant } & \multicolumn{2}{|l|}{ Significant } \\
\hline
\end{tabular}

Table 2 Differences in the ANRAM Data Sets Part 2.

\begin{tabular}{|c|c|c|c|c|c|c|c|c|c|c|c|}
\hline Access points & Count & $\begin{array}{l}\text { Intersecting road } \\
\text { volume }\end{array}$ & Count & Median type & Count & Skid resistance / grip & Count & Street lighting & Count & $\begin{array}{l}\text { Differential } \\
\text { speed limits }\end{array}$ & Count \\
\hline $3 \_4$ & 9 & $4 \_5$ & 9 & $\begin{array}{l}10 \\
-1\end{array}$ & 32 & $1 \_2$ & 5 & $1 \_2$ & 13 & $2 \_1$ & 101 \\
\hline \multirow{2}{*}{$4 \_3$} & 63 & & 9 & & 32 & & 48 & & 13 & & 101 \\
\hline & SRS & & SRS & & SRS & & SRS & & SRS & & SRS \\
\hline $\begin{array}{l}\text { From none / } \\
\text { commercial 1+ }\end{array}$ & 13.3 & $\begin{array}{l}1000 \\
\text { From } 100 / 5,000 \\
1,000\end{array}$ & 61.47 & $\begin{array}{l}\text { From centreline / } \\
\text { central hatching (> } \\
1 \mathrm{~m})\end{array}$ & 11.77 & From medium / good & 17.85 & $\begin{array}{l}\text { From not present / } \\
\text { present }\end{array}$ & 12.8 & $\begin{array}{l}\text { From not present } \\
\text { / present }\end{array}$ & 12.9 \\
\hline $\begin{array}{l}\text { From commercial to } \\
\text { residential } 1 \text { or } 2 \text {, } \\
1+\end{array}$ & 13.1 & & & & & From good / poor & 25.5 & & & & \\
\hline Base SRS & 12.75 & 36.82 & & 12.75 & & 12.75 & & 12.75 & & 12.75 & \\
\hline \begin{tabular}{|l|}
$\%$ \\
SRS \\
\end{tabular} & 4.6 & 66.9 & & -7.7 & & 40.0 & & 0.0 & & 1.3 & \\
\hline$\%$ SRS & 2.5 & N/A & & N/A & & 100.0 & & N/A & & $\mathrm{N} / \mathrm{A}$ & \\
\hline \multicolumn{2}{|l|}{ Not significant } & Significant & & Significant & & Significant & & Not significant & & Not significant & \\
\hline
\end{tabular}


data collected.

Median type: the majority of the changes were found to be code changes of 11 (centreline only) to 10 (central hatching $(>1 \mathrm{~m})$ and these are associated with intersections in which there was an error found in the original coding written to build the MRWA RAP data.

Number of Lanes: The extent of the passing lanes had been modified in the corporate data. It was found that the changing of the number of lanes from one (this is in each direction) to two and one (passing lane configuration) increased the star rating score. Under the iRAP algorithms, head-on crashes are broken into two types: loss of control and overtaking. The number of lanes is an attribute that affects the overtaking head on crash score calculation but is not considered in the loss of control. Increasing the number of lanes to 2 lanes in both directions reduces the star rating score below the original one lane in both direction scores. Using the Star Rating Demonstrator it was found that when the traffic volumes are greater than 6,000 there appears to be a crossover point in the SRS for head on crashes i.e. below this AADT SRS score decreases with the additional of an extra lane.

Differential speeds: in the original data set no allowance for differential speeds between slow moving vehicles was made. In the iRAP coding manual, the following guidance is given "Differential speeds records the difference in either operating speed or speed limit between cars and trucks or cars and motorcycles where it exceeds $20 \mathrm{~km} / \mathrm{h}$." As part of MRWA [5], guidance is given that where vertical grade is $>3 \%$, that the speed differential between cars and slow moving vehicles will be greater than $20 \mathrm{~km} / \mathrm{h}$. The vertical geometry of the road was used to assign the differential speed.

Geometry. A number of changes were found and this is described in more detail below.

\subsection{Differences Found in Road Geometry}

The star rating model and therefore ANRAM is sensitive to horizontal curvature if the curvatures translate into different ratings. The basis of the horizontal curvature rating used for MRWA RAP was the minimum horizontal radius occurring in the $100 \mathrm{~m}$ segment as obtained from Gipsi-Trac (Global and Inertial Positioning System Integration for Tracking Route Alignment and Crossfall) survey of the road. Gipsi-Trac is an ARRB developed product. The Gipsi-Trac data provides continuous (every $10 \mathrm{~m}$ ) geometry information, i.e.:

- horizontal alignment;

- crossfall/ super elevation;

- vertical geometry.

The application of a different technique for selecting the radius will provide a different rating. For example, in New Zealand it is understood that a $30 \mathrm{~m}$ moving average is adopted for obtaining horizontal curvature readings from Gipsi-Trac data which would provide a different curve radius.

As part of the concept design for the proposed treatments (which excludes other projects as explained in the section "Treatments" below), a horizontal alignment was fitted to the existing road centreline using Bentley MXRoad software. In fitting the alignment, there were a number of "kinks" in the alignment that had short curves ( $<5 \mathrm{~m}$ in length) fitted. The results of this alignment was compared to the horizontal geometry obtained from Gipsi-Trac data; in making the comparison the short curves were ignored.

To make the comparison the design chainage had to be converted back to the same linear reference system used for MRWA RAP which is true distance which reflects the actual road length. Refer to Table 3 for a comparison of the two data sets. The comparison only covers the extent for which the concept design was over.

The "Other" records were for the case were there were no matches and these were typically where the extent of the curve may have extended into an adjacent segment. 
Table 3 Comparison of Fitted Geometry Rating Versus Gipsi-Trac data.

\begin{tabular}{lllll}
\hline $\begin{array}{l}\text { Description MX Code } \\
\text { Versus Gipsi-Trac Code }\end{array}$ & Number & Length $\mathbf{( k m )}$ & $\mathbf{\%}$ & $\begin{array}{l}\text { iRAP Code Rating } \\
\text { Approximate } \\
\text { Horizontal Radius }\end{array}$ \\
\hline Match & 139 & 13.9 & 74.7 & $1->900 \mathrm{~m}$ \\
$1-2$ & 20 & 2.0 & 10.8 & $3-200$ to $500 \mathrm{~m}$ \\
$3-2$ & 2 & 0.2 & 1.1 & $2-500$ to $900 \mathrm{~m}$ \\
$2-3$ & 5 & 0.5 & 2.7 & \\
$1-3$ & 8 & 0.8 & 4.3 & \\
Other & 9 & 0.9 & 4.8 & \\
$2-1$ & 2 & 0.2 & 1.1 & \\
$3-1$ & 1 & 0.1 & 0.5 & \\
Total & 186 & 18.6 & 100 & \\
\hline
\end{tabular}

Table 4 Comparison of Fitted Geometry Rating Versus Gipsi-Trac data.

\begin{tabular}{lll}
\hline Category & Star Rating Using MX Geometry & Star Rating using Gipsi-Trac Geometry \\
\hline 1 V 2 & 3 & 2 \\
3 V 2 & 1 & 2 \\
2 V 3 & 2 & 1 \\
\hline
\end{tabular}

Table 5 Comparison of Fitted Geometry Rating Versus Gipsi-Trac data.

\begin{tabular}{llllll}
\hline Description & Run of Road & Head-On & Intersection & Pedestrian (Refer to Note 1) & Other \\
\hline $\begin{array}{l}\text { Preliminary National calibration } \\
\text { factors }\end{array}$ & 0.72 & 0.53 & 0.57 & N/A & 0.61 \\
$\begin{array}{l}\text { Calibration factors developed } \\
\text { for GEH }\end{array}$ & 1.20 & 1.77 & 0.79 & N/A & 0.90 \\
\hline
\end{tabular}

Note 1: Current version of ANRAM does not cater for pedestrians.

In the $1 \mathrm{~V} 2$ category - in the MX analysis the majority of the curves that did not match were close to the change in radius from a 2 to 1 rating which is $900 \mathrm{~m}$. The radii were 900, 905, 915, 980, with the largest radius being 1,050 .

In the 2 V 3 category, it was found that the differences were again close to the change point with the radii being 503, 546, $548 \mathrm{~m}$.

In the $3 \mathrm{~V} \mathrm{2}$, similar again, with curve radii of 500, 515 and $570 \mathrm{~m}$.

Based on the analysis (and assuming that the fitted MX geometry best represents the geometry a driver would select to drive the curves), the use of the minimum radius within the $100 \mathrm{~m}$ segment appears to be conservative.

Horizontal curvature is a significant factor in the star rating scores. To illustrate this point for the same attributes other than horizontal curvature (i.e. the horizontal curvature being the only attribute being changed), the following star ratings are produced for the given differences (this is based on proposed treatment cross section and roadside hazards of trees within $10 \mathrm{~m})$ :

Once the base data had been updated, it was loaded into ANRAM and used to set the basecase, i.e. the highway as it currently is. Using the current crash history of the highway, the model was calibrated using the methodology outlined in ANRAM Calibration Fact Sheet 6 . Ideally the calibration should be undertaken on homogenous sections of the network but sufficient data was not available to do this. Table 5 shows a comparison of the original preliminary national calibration factors versus the derived calibration factors for the Great Eastern Highway.

\section{Treatments}

Within the $40 \mathrm{~km}$ of the highway, there were other projects being considered: one which proposed similar 
treatments as described below and the other a townsite improvement project. The extent of these projects were identified and the ANRAM data for these sections was removed from the updated data set. This was done as cost estimates for these projects were still being developed at the time of the writing of this paper and therefore the economic benefits of these treatments could not be assessed.

Other treatments proposed as part of the upgrade of the highway included Enhanced Information Warning Signs (vehicle activated intersection warning signs that warn drivers on the highway that a vehicle is approaching on the side roads) and other intersection improvements (introduction of left turn pockets and offsetting of the pocket to avoid vehicle masking) but ANRAM cannot assess these treatments.

The following proposed treatments were then assessed:

- Introduction of $1 \mathrm{~m}$ central median - separating one lane in both directions and has audile centrelines. This required widening of the existing seal width to accommodate the median.

- Extension of existing overtaking lanes - not only to increase their length but to improve their start and end locations as a number had poor sight distances. Within the passing lanes, the $1 \mathrm{~m}$ median was carried through although where there were high use accesses within the extent of the passing lanes it was widened to $2.5 \mathrm{~m}$ to provide a defector turning pocket. This widening was thought to reduce the likelihood of high speed rear end crashes where drivers might mistake the right turn indicator being used to signal the turn as an overtaking maneuver in the passing lane.

- Intersection upgrades - provision of left and right turn pockets. Note that ANRAM will only assess right turn pockets (referred to as protected turn lanes).

- Provision of roadside verge barriers at targeted locations (high embankments, to protect a major above ground water main and culverts).

Treatments were assessed individually by the "backdoor" approach in which the codes in the raw data were modified to reflect the treatment being applied. The ANRAM model itself allows users to change the codes directly in the data (using the Toolkit) but one issue associated with using this approach is that there is no traceability in what changes have been made to the data and only three attributes can be changed per treatment.

For the introduction of the $1 \mathrm{~m}$ central median, 4 attributes have to be changed. Median type was changed to wide centerline; centreline rumble strips were set to being present; paved shoulder widths for both driver and passengers sides set to medium (as their width would be $1.5 \mathrm{~m}$ ), etc.

The changes to the data were made by the use of a number of macros written in the visual basic within Excel that used triggers sets in which the user could specify the changes to be made for specific locations or to the entire set depending on if conditions (triggers) were met, e.g. if the median type was centreline only. In addition to making the changes, the macro also wrote into the image reference column of the data the treatment that had been applied to the data. Comparisons of the data before the treatment were applied and after were made to ensure that the changes were made correctly.

The effectiveness of the treatments was assessed on the basis of the crash modification factors and economic analysis using the number of crashes saved. This is described in the sections below.

\subsection{Crash Modification Factors}

Based on the number of predicted crashes compared to the baseline, crash modification factors were determined which were then used to calculate the number of crashes saved and used as the basis of an economic assessment. In determining the crash modification factors, the factor is determined from the ratio of the Predicted FSI crashes (for the treatment) / Predicted FSI crashes obtained from the basecase. ANRAM calculates the predicted and ANRAM FSI crashes at the section level, with a section typically 
being $3 \mathrm{~km}$ in a rural environment and $1 \mathrm{~km}$ in an urban environment. The actual length of each section will depend on the individual segments that are being analysed and sectioning of the data is done as part of the star rating done in ViDA.

\subsection{Economic Analysis}

For the proposed treatments, a cost estimate was developed. In undertaking the modelling, it is important that the costs applied to the ANRAM model match those in the cost estimate. To do this the following process was applied:

1. Using the ANRAM treatment files (these are the files that have the treatments applied to them) determine the total number of $100 \mathrm{~m}$ segments that the treatment has been applied to. If a treatment such as barriers or clearing can be applied to both sides of the segment then the number of segments must reflect this. E.g. If a barrier is applied on passenger and drivers side then the number of segments is counted as 2 . Whereas if the barrier is only applied to one side then the number of segments is counted as 1 .

2. Determine the total cost of the treatment that is applicable. Using barriers as an example the total cost to apply the treatment is the cost of installation, cost of terminals and the cost to remove existing barriers then divide this by the total number of segments for which barriers have been applied.

3. Using the costs determined from Step 2 divide this by total number of segments treated determined in Step 1.

This method provides the following:

- Ensures that the treatment cost applied to the ANRAM matches the cost estimate.

- Takes into account that the treatment lengths in ANRAM do not necessarily match the treatment lengths contained in the cost estimate. This is because ANRAM works on $100 \mathrm{~m}$ segments and therefore as an example if a barrier length was $60 \mathrm{~m}$ as measured in the cost estimate in the ANRAM model it would be applied as $100 \mathrm{~m}$.

In determining the number of crashes saved the crash modification factors determined as outlined above were applied to the ANRAM FSI crashes from the baseline calibrated model. Table 6 contains the indicatives benefits to cost ratio (BCR's) for a 30 year project life using a $7 \%$ discount rate for each individual treatment and then combined treatment with all treatments applied. In determining these BCR's a service life for each treatment was determined and crash costs per crash type for killed and seriously injured was used.

Table 6 Indicative BCR's by Segment and Treatment Type.

\begin{tabular}{|c|c|c|c|c|c|c|c|c|}
\hline \multirow[b]{2}{*}{ Section } & \multirow[b]{2}{*}{$\begin{array}{l}\text { True } \\
\text { Start } \\
\end{array}$} & \multirow[b]{2}{*}{$\begin{array}{l}\text { True } \\
\text { End }\end{array}$} & \multirow[b]{2}{*}{$\begin{array}{l}\text { Section Length } \\
\text { (L) km }\end{array}$} & \multicolumn{4}{|c|}{ Treatment } & \multirow[b]{2}{*}{$\begin{array}{l}\text { All Treatments } \\
\text { Applied }\end{array}$} \\
\hline & & & & $1 \mathrm{~m}$ median & Barriers & $\begin{array}{l}\text { Overtaking } \\
\text { Lanes }\end{array}$ & $\begin{array}{l}\text { Protected Turn } \\
\text { Pockets }\end{array}$ & \\
\hline 5 & 48.50 & 51.50 & 3.00 & 2.2 & 1.6 & 1.9 & 0.5 & 1.7 \\
\hline 6 & 51.50 & 54.50 & 3.00 & 2.2 & 1.7 & 2.9 & 0.7 & 1.9 \\
\hline 7 & 54.50 & 56.70 & 2.20 & 1.9 & 0.5 & 2.2 & - & 2.0 \\
\hline 11 & 67.20 & 69.10 & 1.90 & 1.1 & 0.9 & 1.3 & 0.5 & 1.4 \\
\hline 12 & 70.20 & 73.20 & 3.00 & 0.9 & 0.9 & 1.2 & - & 1.6 \\
\hline 13 & 73.20 & 76.20 & 3.00 & 1.9 & 1.3 & - & - & 2.6 \\
\hline 14 & 76.20 & 79.20 & 3.00 & 1.3 & 0.5 & 1.1 & - & 1.3 \\
\hline 15 & 79.20 & 82.20 & 3.00 & 0.9 & 0.6 & 0.8 & - & 1.1 \\
\hline 16 & 82.20 & 85.20 & 3.00 & 1.1 & 0.8 & 1.0 & - & 1.5 \\
\hline 17 & 85.20 & 88.20 & 3.00 & 1.7 & 0.7 & 1.3 & - & 2.1 \\
\hline \multirow[t]{2}{*}{18} & 88.20 & 88.90 & 0.70 & -0.1 & 0.4 & 0.0 & - & 0.0 \\
\hline & & & Program BCR & 1.5 & 0.9 & 1.4 & 0.6 & 1.7 \\
\hline
\end{tabular}


These are considered indicative as the design is to be further refined, leading to changes in costs and therefore BCR's. At this stage of the work the cost estimates include a significant allowance for contingencies, as well as costs to develop the project (owner's costs) and are based on a probabilistic cost estimating process and show the results based on the P50 (it is expected that $50 \%$ of the time the cost estimate will be exceeded). These BCR's consider safety aspects only. Of particular note is that for the safety barrier treatment, the costs include removal of existing barriers (some of which are not to current standards) and the introduction of $\mathrm{W}$ Beam barriers with closer post spacing to reduce earthworks. For the protected right turn pockets, all of the intersection upgrade cost (which included other intersections improvements, e.g lengthening of left turn pockets as it was difficult to obtain a cost just for the installation of just the protected turn pocket) was used which may account for the lower BCR's being shown.

This was used to prioritise a program of treatments for an interim upgrade of the highway.

\section{Results and Conclusions}

The results of the ANRAM modelling indicates that the proposed treatments will make significant safety improvements to this section of Great Eastern Highway. The star rating of the highway will also be improved.

Using different data sources and applying different rules to data can make significant differences to the star rating scores and therefore the ANRAM outputs as it uses iRAP risk algorithms. If iRAP data is to be generated by methods other than from video, there needs to be protocols developed to provide guidance on governance associated with the data (iRAP requires $10 \%$ to be checked), rules or guidance on how the data can be converted into rating attributes in order for consistent results to be obtained.

Of particular importance, given how much of an effect it has on the star rating scores, are the road condition and skid resistance assessment and guidance is required on how condition data such as roughness, rutting and texture can be translated into iRAP ratings.

\section{References}

[1] Karpinski, J. 2014. "Main Roads trial of Australian National Risk Assessment Model." Presented at 26th ARRB Conference, (ANRAM).

[2] iRAP. 2014. "Star Rating and Investment Plan Coding Manual Drive on the left edition." Accessed June 2014. http://downloads.irap.org/docs/RAP-SR-2-2_Star_Rating _coding_manual.pdf.

[3] iRAP. 2014a. "Data Load Specification." Accessed June 2014. http://vida.irap.org/en-gb/home.

[4] ANRAM. 2014. "Calibration Fact Sheet 6. Accessed June 2014. https://www.arrb.com.au/admin/file/content2/c7/A NRAM_FactSheet6.pdf

[5] MRWA. 2011. Policy and Guidelines for Overtaking Lanes Document No. D11\#321098. Accessed June 2014. https://www.mainroads.wa.gov.au/BuildingRoads/Standa rdsTechnical/RoadandTrafficEngineering/GuidetoRoadD esign/Pages/MRWA_Supplement_to_Austroads_Guide_t o_Road_Design___Part_3. aspx. 